\title{
INVESTIGACIONES
}

\section{Violencias Simbólicas en el Trabajo Docente*}

\author{
Symbolic Violence in Teaching Work
}

\author{
Pamela Barría Herrera $^{a}$, Christian Miranda Jaña ${ }^{b}$, \\ María Isabel Toledo Jofréc \\ ${ }^{a}$ Universidad Metropolitana de Ciencias de la Educación, Chile. \\ pamela.barria@umce.cl \\ ${ }^{b}$ Universidad de Chile, Chile. \\ christian.miranda@u.uchile.cl \\ ${ }^{c}$ Universidad Diego Portales, Chile. \\ maria.toledo@udp.cl
}

\section{RESUMEN}

El estudio describe las violencias simbólicas que experimentan profesores que trabajan en tres escuelas de dependencia municipal, particular-subvencionada y particular-privada de la Región Metropolitana. La metodología es de tipo cualitativa, con uso de observaciones y entrevistas, cuyos datos son analizados mediante el procedimiento de descripción densa. Los resultados develan la presencia de violencias simbólicas asociadas al control del tiempo, del cuerpo y de la acción pedagógica, observadas a partir de la interacción de elementos como el tipo de administración de los establecimientos educacionales, sus normativas laborales y la cultura escolar.

Palabras claves: Violencia Simbólica, Cultura Escolar, Administración Educativa, Normativas Laborales, Trabajo Docente.

\section{ABSTRACT}

This study describes the symbolic violence experienced by teachers who work in three different types of schools -public, private-subsidized, and private schools- in the Región Metropolitana, Chile. A qualitative approach was used to analyze observations and interviews through thick description. The results show the presence of symbolic violence associated with the control of time, body, and pedagogical action, observed in the interaction of elements such as the type of school administration, labor regulations, and school culture.

Key words: Symbolic violence, school culture, school administration, labor regulations, teaching work

* Estudio financiado por CONICYT-Chile y realizado en el marco de los Proyecto Fondecyt $\mathrm{N}^{\circ} 1140135$ y 1170328. 


\section{INTRODUCCIÓN}

La labor docente corresponde a un conjunto de tareas y relaciones humanas estructuradas de determinadas maneras, ubicadas en la escuela como espacio físico (Hargreaves, 2003). En Chile, se desarrolla en un contexto marcado por reformas que implican un cambio del modelo con consecuencias para el trabajo del docente (Andrade, 2006). Desde 1981 comienza la descentralización del sistema educativo, basado en la privatización, por medio del cual las escuelas estatales son gestionadas a nivel municipal por una figura intermediaria denominada sostenedor, quien recibe y administra los recursos del Estado y se promueve el desarrollo de escuelas particulares subvencionadas. Desde entonces, los establecimientos escolares operan bajo tres tipos de administración: escuelas gestionadas por el municipio; particulares subvencionadas, gestionadas por sostenedor privado con subsidios estatales y las particulares privadas, gestionadas por un sostenedor privado sin subsidio estatal. Además, se instala un mecanismo de financiamiento basado en un sistema de voucher o subsidios portables a la demanda, en los que los recursos dirigidos hacia la escuela dependen del número de estudiantes y su asistencia al establecimiento educacional (Cornejo et al., 2015).

En los años noventa, se desarrollan reformas en las que se incorpora un modelo educativo basado en mecanismos de mercado y competencia, sistema al que se han introducido progresivamente elementos de enfoques gerencialistas o del New Public Management. Este modelo de gestión escolar promueve la contratación flexible, la estandarización de resultados esperados y de procesos de trabajo, la rendición de cuentas, la evaluación individual y los ajustes de salarios de los trabajadores asociados a resultados, el fomento de la competencia individual entre trabajadores, los procesos de marketing en el sistema educativo y la clientelización de sus participantes (Cornejo et al., 2015).

Las regulaciones bajo este modelo repercuten directamente en la composición, estructura y gestión de las redes públicas de enseñanza. A partir de su implementación se generan medidas que alteran la configuración de las escuelas en sus aspectos físicos y organizacionales, concibiendo adecuaciones según los criterios de productividad, eficacia, excelencia y eficiencia presentes en los programas de reforma, las que además están marcadas por la estandarización de procesos administrativos y pedagógicos, que permiten reducir costos, redefinir los gastos y garantizar el control centralizado de las políticas (Andrade, 2006).

La intensificación laboral se presenta como una consecuencia del modelo. Este proceso implica la erosión en los privilegios laborales, consistente en la reducción de tiempo de descanso en la jornada laboral, la carencia de tiempo para fortalecer los saberes profesionales y mantenerse al día en su campo, la sobrecarga que reduce las áreas personales, inhibe la participación en la planificación a largo plazo y el control sobre la misma favorece la dependencia a materiales producidos fuera, conduce a la reducción de la calidad de servicio y, por último, conlleva a la diversificación de la pericia y la responsabilidad para cubrir la falta de personal. Además, muchos profesores han confundido la intensificación con la profesionalidad (Hargreaves, 2003). Así, la auto intensificación y la imposibilidad de responder a todas las demandas son fuente de sufrimiento, insatisfacción, enfermedad, frustración y fatiga (Oliveira, Goncalves y Melo, 2004).

Por otro lado, a partir de la perspectiva de la labor docente como un trabajo, es posible identificar formas de regulación del quehacer. Las normativas laborales se establecen como la constitución de derechos, libertades, garantías para el ejercicio y principios básicos 
que orientan la acción de poderes públicos en lo político, económico y social. Además, dicta normas básicas de legislación laboral, disposiciones sectoriales, normas orgánicas procedimentales y ejerce potestad reglamentaria, todo lo anterior planteado desde la función normativa del Estado (Köhler y Artiles, 2010). De acuerdo a ello, los marcos reguladores que contemplan el trabajo docente en Chile en establecimientos de dependencia municipal corresponden al Estatuto Docente (ED), el cual tiene vigencia desde 1997 hasta el año 2016, ya que en abril de ese año se instaura el Sistema de Desarrollo Profesional Docente. Otra normativa de trabajo corresponde al Código del Trabajo como marco general. Estas normativas conviven con aquellas regulaciones dadas en la cultura escolar de cada establecimiento educacional.

El ED rigió a profesionales de la educación, cargos directivos y técnico pedagógicos en Departamento de Administración Municipal, establecimientos de educación primaria y secundaria, de administración municipal o particular reconocida oficialmente, también en los establecimientos de educación parvularia subvencionados y de educación técnicoprofesional administrados por corporaciones privadas sin fines de lucro. En él se describen las áreas de trabajo de profesionales de la educación, normas, requisitos, deberes, obligaciones y derechos de carácter profesional (Mineduc, 1997). El ED es el marco regulador vigente al momento de desarrollar el proceso de producción de datos de este artículo.

Entre sus artículos, define la función docente con carácter profesional de nivel superior, a cargo de los procesos sistemáticos de enseñanza y educación. Entre sus tareas se distingue la docencia de aula -acción o exposición personal directa realizada en forma continua y sistemática- y actividades curriculares no lectivas -labores y actividades educativas complementarias de la función docente de aula-. Su distribución semanal se considera a la base de un máximo 44 horas: quienes cuenten con jornada completa, la docencia de aula no debe exceder de 33 horas, excluidos los recreos, el horario restante es destinado a actividades curriculares no lectivas. En los establecimientos educacionales con Jornada Escolar Completa Diurna, quienes cuentan con 44 horas en su contrato no podrán exceder 32 horas con 15 minutos, excluidos los recreos, y el horario restante es destinado a actividades curriculares no lectivas. Para ambos casos, para los contratos inferiores a 44 horas semanales, el máximo de clases se determina por la proporción respectiva (Mineduc, 1997).

Además, el ED describe en la dotación docente como profesores en calidad de titulares o en calidad de contratados. Los primeros se incorporan a una dotación docente previo concurso público de antecedentes, mientras quienes están en calidad de contratados, desempeñan labores docentes transitorias, experimentales, optativas, especiales o de reemplazo de titulares (Mineduc, 1997).

Con la vigencia el Sistema de Desarrollo Profesional Docente (SDPD) se generan cambios al ED en el ámbito formativo, laboral y profesional. Entre ellos, la acreditación obligatoria y evaluación en la formación inicial docente, un proceso de inducción para docentes principiantes quienes son acompañados por mentorías, gratuidad en cursos de formación permanente, definición de una carrera docente basada en tramos profesionales, incentivos económicos basados en el desempeño y la experiencia, entre otros (Mineduc, 2016). Cabe destacar que esta nueva legislación explicita la responsabilidad de directores sobre la asignación de tareas para que las horas no lectivas sean efectivamente destinadas para ello; es más, se agrega al artículo $8^{\circ}$ la consideración que "Los docentes que vean vulnerados los derechos antes descritos podrán ejercer las acciones legales que sean procedentes." (Mineduc, 2016, p. 3). 
Simultáneamente, el Código del Trabajo regula el trabajo docente en Chile, específicamente en establecimientos educacionales particulares privados y particulares subvencionados. Entre sus puntos, reglamenta las relaciones laborales entre los empleadores y los trabajadores que no están sometidos por ley a un estatuto especial, como es el caso del ED y/o SDPD. Entre sus artículos, destaca que las relaciones laborales deben fundarse en un trato compatible con la dignidad de la persona, siendo contrario - entre otras conductasel acoso sexual, acoso laboral y actos de discriminación. Además, presenta regulaciones como la definición y consideraciones de jornada de trabajo, tiempo de colación y define remuneración como el sueldo, el cual debe dar cumplimiento al principio de igualdad de remuneraciones entre hombres y mujeres (Dirección del Trabajo, 2015).

En la misma línea, ED y la Ley Calidad y Equidad de Educación (Mineduc, 2011), abordan el derecho de los profesionales de la educación a trabajar en un ambiente de tolerancia, de respeto mutuo a su integridad física, psicológica y moral, no pudiendo ser objeto de tratos vejatorios, degradantes o maltratos psicológicos por parte de los demás integrantes de la comunidad educativa. En consideración a lo anterior, reviste especial atención todo tipo de violencia física o psicológica cometida por cualquier medio, en contra de los profesionales de la educación.

Como se señala previamente, otra forma de ordenamiento que vive la escuela en su mundo interno corresponde al concepto de cultura escolar, proceso fundamental que explica cómo funcionan las acciones rutinarias propias de una institución (Falsarella, 2018). De acuerdo a lo planteado por Forquin (1993, en Falsarella, 2018) la cultura escolar implica la producción y gestión de símbolos, ritos y lenguajes, así como a los modos de regulación y transgresión establecidos. En la misma línea, Deal \& Peterson (2009) identifican reglas y rituales -éstos últimos accesibles de manera implícita-, los cuales permean aspectos como la actuación, vestimenta, habla y apoyos; en caso de no seguirlos, conllevarían sanciones sociales.

La cultura escolar demuestra la fuerza de las tradiciones y la complejidad de realizar cambios en ella, exponiendo el difícil equilibrio entre la reproducción e innovación. En la escuela no existe un vacío social; su estudio permite comprender las relaciones entre la educación escolar y otras formas sociales de formación de los sujetos, en particular, las relacionadas con formas de ejercicio del poder en la sociedad (Falsarella, 2018).

$\mathrm{Al}$ describir el trabajo docente chileno, los resultados de TALIS 2018 exponen que el $15 \%$ del profesorado está "de acuerdo" o "muy de acuerdo" con la valoración social de la profesión, mientras que el promedio de países de la OCDE corresponde a un 26\%. Para el caso de la satisfacción con los términos del contrato laboral, 54\% del profesorado está de acuerdo con ello (promedio OCDE de 66\%) y con respecto a los sueldos, el 38\% plantea estar satisfechos, lo que no difiere significativamente de los promedios alcanzados por países OCDE, correspondientes a un 39\% (OECD, 2020.)

En la misma línea, el año 2013 el Ministerio de Educación (2013) proporciona datos respecto a las condiciones contractuales de profesores en Chile, en las cuales dichos actores declaran que el $69 \%$ tiene jornada completa y el $63 \%$ tiene contrato indefinido. Ahora bien, el informe TALIS 2018 expone que el 62\% de los y las docentes tienen contrato permanente (promedio OCDE 82\%), mientras que un 22\% están empleados con contratos de un año o menos (promedio OCDE 12\%). Estos valores exponen la estabilidad de las condiciones contractuales durante el periodo señalado (OECD, 2020.)

Si bien las normativas laborales abordan el derecho a trabajar en un ambiente de tolerancia y respeto mutuo, la evidencia da cuenta de la existencia de violencias hacia los 
docentes en su contexto laboral. Sin embargo, lo escasamente reportado en la literatura sobre la violencia que viven los docentes, solo se describen interacciones violentas entre directivos y docentes (Blasé \& Blasé, 2008) y entre colegas (Ortiz-Molina, 2011; Morales et al., 2014). En el caso de Chile, predominan declaraciones de violencia de carácter psicológico, sin diferencias significativas según género (Mineduc, 2005), elemento coincidente con estudios en Colombia (Ortiz-Molina, 2011), que recalcan la necesidad de observar las condiciones laborales que se presentan en el trabajo docente, como una manifestación de los tipos de violencias presentes en las escuelas.

Los antecedentes anteriormente descritos refieren a la presencia de violencias en el trabajo laboral. Ahora bien, definir violencias resulta una tarea compleja, pues existe diversidad de perspectivas para abordarla (García y Madriaza, 2005; Berger y Lisboa, 2012), múltiples tipos de violencias, que varían en intensidad de sus prácticas, finalidades y naturaleza; además es relativa, según épocas, medios sociales y culturales. De esta forma será necesario hablar de "las violencias" y no de "la violencia" (Crettiez, 2009).

Los antecedentes descritos evidencian la presencia de violencia simbólica, comprendida en este estudio como un tipo de violencia ausente de dolor visible que presenta un discurso hegemónico que lo legitima. Es un fenómeno culturalmente validado, el cual opera bajo un mecanismo de "servidumbre voluntaria" (Crettiez, 2009). De acuerdo a Bourdieu y Wacouant (1995), esta forma de violencia se ejerce sobre un agente social con su anuencia. En términos estrictos, los agentes sociales son conscientes de que, aunque estén sometidos a determinismos, contribuyen a producir la eficacia de aquello que los determina, en la medida en que ellos lo estructuran. El efecto de dominación casi siempre surge durante los ajustes entre los determinantes y las categorías de percepción que los constituyen como tales.

Bourdieu y Wacouant (1995), abordan el desconocimiento como el hecho de reconocer una violencia que se ejerce precisamente en la medida que se le desconoce como tal; se acepta ese conjunto de premisas fundamentales, pre-reflexivas, que los agentes sociales confirman al considerar al mundo como autoevidente y natural porque le aplican estructuras cognoscitivas surgidas de la estructura misma de dicho mundo. Al nacer dentro de un mundo social, se aceptan algunos postulados y axiomas, los cuales no se cuestionan y no requieren ser inculcados. Por esta razón, el análisis de la aceptación dóxica del mundo, que resulta del acuerdo inmediato de las estructuras objetivas con las estructuras cognoscitivas, es fundamento de una teoría realista de la dominación y de la política. De esta forma, esta violencia se desarrolla al ser ejercida simplemente por el orden normal de las cosas. El Estado, las instituciones y las prácticas del orden dominante tienden a ocultar, bajo aspecto de naturalidad, relaciones dominantes invisibles (Crettiez, 2009).

Resulta relevante abordar el fenómeno de las violencias simbólicas desde la subjetividad de sus actores, ello ante una brecha de conocimiento respecto a lo que sucede en el trabajo docente en la escuela. En efecto, según Hargreaves (2003), las violencias son un factor que afecta la calidad de vida laboral y el desarrollo de la enseñanza, por lo que analizarlas constituyen un aporte al trabajo docente, asumiendo que la construcción de un buen trato en la comunidad escolar involucra a todos quienes son actores de la escuela, incluyendo en ello a profesionales de la educación. Así, se define como objetivo del estudio describir violencias simbólicas que sufren profesores en tanto trabajadores de la educación.

Este trabajo se estructura a continuación en torno a los siguientes ejes de desarrollo. El primero, aborda el diseño metodológico que guía la producción de datos del fenómeno de la violencia en las normativas laborales. El segundo, presenta una síntesis de los principales 
hallazgos del estudio y su interpretación. El tercero, da cuenta de la discusión de resultados y conclusiones finales, focalizando en las implicancias educativas del estudio.

\section{METODOLOGÍA}

La investigación se sitúa desde un método cualitativo con uso del enfoque interpretativo, ya que busca analizar el fenómeno de las violencias en las normativas laborales en la escuela desde la experiencia social de las y los propios actores (Denzin y Lincoln, 2012).

El estudio selecciona un total de tres establecimientos educacionales, cada uno de los cuales refieren a uno de los tipos de administración: municipal (EM), particular subvencionada (PS) y particular pagada (PP). Además, se consideraron como criterios de elección su ubicación en 3 comunas diferentes de la zona urbana de la Región Metropolitana. Es coincidente entre los establecimientos educacionales, brindar educación parvularia y primaria, además de su carácter mixto -acceden niños y niñas-. En el caso de los sujetos, el estudio cuenta con un total de 18 profesores con antigüedad mínima de 1 año en el establecimiento educacional. Se incluyen 7 docentes con cargos directivos dada su relevancia y participación en el tema. Su participación contempla la voluntariedad, confidencialidad y conocimiento del estudio, a partir de la firma de consentimientos informados.

En cuanto a las particularidades de los establecimientos, en el caso de la escuela municipal, ésta brinda educación a cerca de 500 estudiantes de párvulos y primaria. Cuenta con un promedio de 27 estudiantes por aula, donde ejercen un total de 30 profesores. Las relaciones laborales entre sostenedor y docentes que tienen contrato vigente se regulan desde el ED. En el caso del establecimiento de dependencia particular subvencionado, es gestionado por un sostenedor privado con subsidios estatales. Atiende a más de 900 estudiantes, desde párvulos a educación secundaria. Cuenta con un promedio de 30 estudiantes por curso y un total de 53 docentes. Las relaciones laborales entre los sostenedores y docentes se rigen por las normas del Código del Trabajo y sus disposiciones complementarias en todo aquello que no esté expresamente contenido en el ED. Por último, el establecimiento de dependencia privada atiende a más de 1000 estudiantes distribuidos en tres ciclos escolares: parvularia, primaria y secundaria, con un promedio de 24 estudiantes por curso y un total de 54 docentes. Las relaciones laborales entre sostenedor y docentes se rigen por el Código del Trabajo.

El proceso de producción de datos se desarrolla mediante observaciones y entrevistas, considerando como criterios de selección la voluntariedad de los sujetos y accesibilidad de los establecimientos ya antes descritos. Así, se realizaron 5 observaciones en reuniones de trabajo como el Consejo de profesores o instancias similares, cuyo registro se realiza por medio de notas de campo y audio; debido al acceso a los espacios, 4 fueron realizadas en el establecimiento municipal y una de ellas en el privado. En el caso de las entrevistas, se efectúan un total de 25 , de las cuales 11 corresponden a la escuela municipal, 5 en el establecimiento particular subvencionado y 9 en el colegio particular privado; todas ellas registradas por medio de grabadoras de audio y luego transcritas a texto.

Los datos recogidos se analizan por medio de descripción densa, proceso por el cual se encara una multiplicidad de estructuras conceptuales complejas, muchas enlazadas o superpuestas entre sí, en donde el investigador debe ingeniárselas para captarlas y luego explicarlas (Govea, Vera y Vargas, 2011). 


\section{RESULTADOS}

Como resultados del estudio se identifican acciones vinculadas a las normativas laborales y la cultura escolar de los distintos establecimientos participantes, los cuales serán descritos a continuación.

\subsection{NORMATIVAS LABORALES}

En el establecimiento de dependencia municipal, quienes trabajan allí presentan distintos tipos de vinculaciones contractuales. La primera de ellas corresponde a aquellos profesionales contratados como titulares o planta, quienes presentan continuidad en sus contratos año a año, no así en la cantidad de horas dispuestas. En el segundo lugar se encuentran aquellos profesores en modalidad "contrata" y presentan vinculación con su empleador durante el año escolar, sin embargo, no cuentan con la seguridad de continuidad al año siguiente. Quienes están en esta modalidad contractual, son informados en diciembre de cada año si continúan el año siguiente, de no ser así, son avisados en marzo, es decir, al inicio del año escolar próximo. En esta categoría hay profesores con años de contrato en la comuna, sin embargo aún no cuentan con titularidad esperada, lo que es atribuido a la organización administrativa. Por último, se presentan aquellos profesionales contratados bajo la Ley de Subvención Escolar Preferencial [Ley SEP]. En esta modalidad contractual, no cuentan con la certeza de continuidad año a año. De este modo, para las tareas solicitadas en el establecimiento educacional, se presentan tres modalidades contractuales distintas.

La continuidad laboral en el establecimiento municipal se debe principalmente a dos factores: el primero de ellos refiere a la Ley 20.501, la cual señala que son los directores quienes definen las plantas docentes de cada escuela. Otro elemento corresponde a la demanda de matrículas, constituyéndose como un elemento de preocupación para quienes trabajan en este tipo de establecimiento. Por ello, en la escuela se realizan campañas municipales de captación de matrículas, lo que afecta en la continuidad de tareas de carácter educacional al irrumpir actividades: "La gestión de las horas de los profesores es violenta. Se crea incertidumbre sobre la situación laboral de los titulares y los a contrata" (MOB) ${ }^{1}$.

En el caso del establecimiento privado, sus actores señalan estar sujetos a un contrato de prestación de servicios. Como instancia privada que tiene que responder a un servicio definido, se pueden ver enfrentados a demandas de carácter jurídico por incumplimiento de contrato desde sus apoderados. La actitud de estos últimos frente a este contexto se señala en las siguientes citas:

Este contrato de prestación de servicio... es como: yo quiero mi cuarto de libra ahora; es como yo estoy pagando y quiero que el servicio sea el óptimo (PO).

En este tipo de colegios privados también uno siente la violencia desde algunos apoderados que sienten que están comprando un producto y si no les satisface tienen que reclamar como si fueran a un supermercado. Y eso, los profesores sentimos esa violencia (PCC).

Para identificar al tipo de entrevistado, se utiliza la siguiente fórmula: (Instrumento - Tipo de Establecimiento). Se utiliza ENT para entrevista y OBS para observación. EM para establecimiento municipal, PS para establecimiento particular subvencionado y PP para establecimiento particular pagado. 
Respecto a la jornada laboral, la distribución de las horas de trabajo se divide entre aquellas que son de docencia en aula y aquellas actividades curriculares no lectivas, es decir, labores educativas complementarias a la función docente de aula relativa a los procesos de enseñanza-aprendizaje. La distribución de estas horas en el colegio privado corresponde, en el caso de profesores de primaria, a un contrato cuya jornada tiene 32 horas frente al curso y 13 horas de permanencia, aspecto considerado adecuado por sus actores. Sin embargo, en el caso de profesores se secundaria tienen en su contrato solo aquellas horas destinadas para el trabajo directamente en aula, "Tienen 42 horas... son 42 horas frente al curso" (ENT PS).

Para quienes son profesionales de la educación, el tiempo destinado para realizar tareas asociadas a su rol se consideran como insuficiente, lo que se ejemplifica en la planificación y en la posibilidad de capacitarse. Lo anterior se expone en la siguiente cita:

Hacemos cosas bien, también hacemos cosas mal, cuando yo pienso que hacemos cosas mal, no es que queramos hacerlas mal porque, por ejemplo, a mí en la Agencia de Calidad, las planificaciones... piden todas, pero en qué momento el profesor planifica...si el profesor no tiene el tiempo suficiente para poder planificar... o capacitarse (ENT EM).

Quienes son trabajadores de la educación en el colegio privado, deben realizar registro de entrada y salida a las jornadas de trabajo por medio de máquinas que verifican huella digital. Este registro se realiza en el ingreso al establecimiento, finalización de actividades, así como en los tiempos destinados al almuerzo. Su implementación tiene impacto en el desarrollo del trabajo y en las remuneraciones, debido a que se podría producir retraso en el ingreso ante las características físicas del establecimiento educacional y la cantidad de máquinas disponibles para el registro:

Vamos a estar todos haciendo la fila en la maquinita y no sé si vamos a alcanzar a tomar la clase a las 2:10 pero se supone que si tú no tomas la clase a las 2:10 hrs, te van a descontar por no ser puntual en tu inicio... El problema es que hay pocas máquinas, esta acá y otra que está allá, en el parvulario, está más lejos (ENT PP).

Dentro de la jornada laboral se identifica el uso del horario de almuerzo. En el colegio privado se comenta la implementación de una directriz interna, en la cual se utiliza parte del horario de colación del docente con acciones que se orientan al cuidado de estudiantes, pues cada profesor debe estar en sala hasta que se retira el último estudiante. Esta normativa tiene efectividad durante un periodo limitado de tiempo ya que se deja de ejecutar por sus profesores. En cambio, en el establecimiento de municipal se visualiza la implementación de la política educativa de Jornada Escolar Completa como un factor que afecta en el horario dispuesto para almuerzo. Es por ello que se amplía el espacio destinado para comer, de media hora a cuarenta minutos.

En horario de almuerzo...optaron la medida de que no podíamos salir de la sala hasta que saliera el último alumno del aula y eso implica que me quedo mucho rato... entonces ya implicaba tener menos tiempo para tu hora de almuerzo... era una complicación tremenda, no sé, habrá durado tres semanas y después ahí ya nadie más la cumplió (ENT PP). 


\subsection{CULTURA ESCOLAR}

Dentro de las regulaciones brindadas a partir de la cultura escolar de los establecimientos estudiados, se identifica el uso del uniforme en los y las trabajadores de la educación. En el caso del colegio privado se hace uso del delantal; si bien se plantea que es para todos quienes forman parte del colegio como medio de presentación personal, se menciona la posibilidad de elección para quienes son parte de las coordinaciones. De igual manera se identifica resistencia en el uso en el caso de profesionales jóvenes.

Todos deben usarlo. Creo que algunos jóvenes o profesores se resistían, pero también es parte de la presentación personal. Lo que más te formaliza es el delantal... Como coordinadora podría no usarlo, pero soy profesora básica, entonces siempre he creído que si paso por el patio con mi delantal y hay alguien que necesita ayuda y lo recojo, si me ve con el delantal, siente que soy alguien de confianza... Eso es un tema personal mío, porque en general algunos directivos andan sin delantal (ENT PP).

En el caso del colegio subvencionado, también se menciona el uso de delantal en la presentación personal. Este uniforme es entregado por el colegio y pagado por los y las profesionales. En el caso del establecimiento municipal, se observa el uso de delantal en las profesoras y en las asistentes de la educación, mientras quienes ocupan cargos directivos no hacen uso de este tipo de uniforme. Se hace hincapié al uso del delantal en actividades protocolares, haciendo énfasis en su uso por parte de las mujeres.

Respecto a la labor pedagógica, en el establecimiento subvencionado se definen tareas formales que cuentan con un cronograma para su cumplimiento: tener planificaciones, pruebas a ciertas fechas, revisiones, cierta cantidad de notas en fechas definidas. Se suman a estas tareas la contención al grupo de estudiantes y tener dominio del aula que, de acuerdo a lo comentado, en algunos cursos es más complejo que en otros. Además deben tratar con apoderados, cuya relación es considerada violenta, ya que exigen por pagar.

La planificación clase a clase es una tarea que en el establecimiento municipal se describe como una exigencia que no se puede cumplir debido al tiempo que requiere para efectuarse. Además, no encuentran sentido a la acción en un año escolar de constantes cambios. De igual manera, se describen a los y las profesores agobiados, debido a que los aprendizajes de sus estudiantes son más lentos a lo solicitado desde el Ministerio del ramo. Lo anterior se describe en la siguiente cita:

A veces los aprendizajes son más lentos de lo que pensamos, entonces por el lado el Ministerio dice: para que el niño pase de curso tiene que tener tales logros, pero a veces los tiempos no dan porque tú tienes un ritmo de aprendizaje que es distinto a este otro (ENT EM).

La escuela se define como una entidad que se ha transformado, debiendo responder a mucha burocracia. En el caso del establecimiento municipal, específicamente en el Proyecto de Integración Escolar, se utiliza la mitad del tiempo para redactar informes más que para desarrollar actividades propias del apoyo al aula y aprendizaje de estudiantes. Otro ejemplo es la exigencia de tener documentado en protocolos, de manera sistemática 
y al día, aquello que anteriormente se hacía en la escuela, pero no estaba escrito. Estos protocolos pueden ser solicitados en supervisiones.

Hay organizaciones que supervisan la escuela, entre ellas la Agencia de Calidad de la Educación, Superintendencia de la educación, Subvenciones e Infraestructura. En el caso de la Agencia de Calidad, su supervisión es un trabajo integral, quedándose un mínimo de tres días en la escuela para hacer observación. Se recalca desde la escuela municipal que es un trabajo preventivo. Luego de la supervisión entregan una pauta de sugerencias que hay que arreglar como institución; con posterioridad toman esa pauta y van a evidenciar si se hicieron o no los cambios planteados. Si bien desde dirección se define que no es punitiva, señalan que encasilla a la escuela. A la fecha de observación, se encontraban en periodo de "marcha blanca".

Otra organización que supervisa a la escuela es la Superintendencia de Educación. Su visita, que en la escuela municipal se menciona como reiterada en el año, genera tensión y presión constante, lo que se expone en la siguiente cita:

Su visita...tensiona mucho y si vienen como a ver que tú estés, ya te están tratando mal antes de haberte visto y diciéndote "usted no puede cometer errores... En el año vienen por lo menos cuatro veces, entonces de repente hay que tener todos los libros al día, los documentos... dicen van a venir y esa semana hay que tener todo listo, hay una presión constante ahí y hay que rendir (ENT EM).

También acude a la escuela la supervisión de las subvenciones. Para esta inspección se explicita que la asistencia de estudiantes es el punto de inflexión, la cual se registra en el libro de clases por medio de un punto si el estudiante está presente. Esta fiscalización es definida como punitiva, debido a que ante el error se cursan multas consideradas altas. Además, también se realizan supervisiones a la infraestructura, inspeccionando toda la edificación, incluyendo infraestructura y aseo.

Entonces hay cosas que son muy rígidas, como le pides tú a un ser humano que no cometa un error... la subvención ven la sala cuántos niños hay presentes y cuántos ausentes entonces estás pasando la lista, pones un puntito si está presente, y te equivocas o hiciste todo bien y hay que hacer una sumatoria, la gente se equivoca de repente cuando suma... y eso es multa, y la multa la paga el municipio, multas grandes (ENT EM).

En términos generales, en la escuela municipal refieren a las supervisiones como fuentes de tensión desde la base del control y de la interpretación de dolo de ante las faltas de los actores de la escuela desde quienes supervisan. Además, las distintas instancias que inspeccionan solicitan cada vez más informes y protocolos para diversas acciones, siendo una falta grave la no documentalización en una cultura escolar que opera desde lo vivencial. Las consecuencias de las supervisiones también impactan desde Dirección hacia docentes, pues si no hay cumplimiento de las tareas, se llamará al director, jefe de unidad técnico-pedagógica y profesor, "manchando" la hoja de vida del docente por su falta.

Control. Mucho control... Por lo tanto, si hablo del control, no es porque haga las cosas mal, lo que pasa es que faltan algunos elementos dentro de esta cadena, que 
necesitan ser llenados"... “Cualquier cosa, la superintendencia te la interpreta como que es "dolo" como que tú quieres engañar al Estado, y como afecta eso, la impresión que nosotros tenemos que la gente que viene a supervisarte en ese aspecto, viene pensando que tú has hecho algo indebido, viene predispuesto, esa es la impresión que tiene uno, de pillarte en algo, entonces indudablemente eso te tensiona (ENT EM).

Por último, la Evaluación docente es manifestada en el colegio municipal como fuente de violencia y estrés debido a la forma en la que se realiza, ya que no se destinan tiempos para prepararla, lo que afecta en la vida personal al contar con menos horas de sueño y descanso. Estas impresiones se describen por medio de la siguiente cita:

Provoca cierta violencia y que nos provoca estrés... yo estoy de acuerdo que haya una evaluación porque es necesario... ver cuáles son nuestros conocimientos, nuestros manejos, pero a lo mejor la forma no es adecuada, porque no nos dan los tiempos para hacerlo, también es tiempo extra que tenemos que ocupar para poder desarrollar esta evaluación y significa menos horas de sueño, de descanso...entonces para mí eso es una forma también de violencia hacia nosotros (ENT EM).

\section{ANÁLISIS}

A partir de los resultados descritos en torno a las dimensiones normativas laborales y cultura escolar, se plantea que, en el primer aspecto se presentan variadas manifestaciones de violencia en el trabajo docente: distintos tipos de contrato para efectuar labores similares, incertidumbre ante la continuidad laboral, un limitado uso efectivo del trabajo no lectivo, el registro de los tiempos de trabajo en condiciones descontextualizadas a las características del colegio y la ocupación de tiempos considerados para el almuerzo. De este modo, resulta coincidente que la mayor parte de las violencias descritas se presentan desde el control del tiempo de quienes son trabajadores de la educación.

Se identifica la ocupación del tiempo personal de profesores para el desarrollo de acciones laborales; ello puede ocurrir tanto dentro de la escuela, como el uso de los tiempos de almuerzo, así como también fuera del espacio físico del establecimiento educacional, ante la invisibilización de los tiempos destinados para las actividades curriculares no lectivas, entendidas como aquellas labores educativas complementarias de la función docente de aula, como tareas de jefatura de curso, administración de la educación; actividades anexas o adicionales a la función docente propiamente tal, actividades coprogramáticas y culturales; actividades extraescolares; actividades vinculadas con organismos o acciones propias del quehacer escolar, entre otras (Mineduc, 1997), acciones que resultan indispensables para la concreción de una clase y el desarrollo educativo de los estudiantes.

A ello se suma la burocracia como manifestación de desconfianza ante el quehacer del docente en sus tiempos de trabajo. Sin embargo, para efectuar las practicas burocráticas como el uso de registros de entrada y salida, no se consideran las realidades contextuales con las que cuenta el colegio. Es por ello que estas medidas se perciben con el riesgo que afecten en sus remuneraciones y en los tiempos para ingresar al establecimiento. Ahora bien, se hace la distinción en el caso del colegio municipal, donde la normativa externa, como la Jornada Escolar Completa, es adaptada para favorecer la permanencia de sus profesores en el colegio. 
Por otro lado, se identifican distintos tipos de contrato: en el establecimiento municipal se definen tres tipos de contrato para realizar labores similares: titular o planta, contrata y bajo Ley SEP. Estas tres modalidades presentan diferencias significativas en la percepción de continuidad laboral, ya que solo la titularidad lo tiene asegurado, no así la continuidad de sus horas de contrato de un año a otro. Este contexto es distinto a quienes están en modalidad contrata y bajo Ley SEP, ya que son informados año a año si continúan trabajando en el establecimiento, ya sea a finales del año escolar o a puertas del siguiente. De este modo, quienes son actores definen la gestión de horas como violenta, siendo participes de actividades de promoción para la captación de matrículas, con ello favorecer uno de los factores que contribuyan a mantener su puesto de trabajo.

En el caso del colegio privado, la presencia de un contrato de prestación de servicios afecta en la relación entre profesor-apoderado y en el desarrollo del mismo trabajo docente. Lo anterior se debe a la visualización del apoderado en la exigencia de un servicio en estatus similar a una compra de un bien y la vulnerabilidad del docente ante la posibilidad de demandas de carácter judicial ante la ejecución de su trabajo.

En la dimensión cultura escolar, en primer lugar, es coincidente en los tres colegios el uso de uniforme en los profesionales de la educación para la presentación personal, específicamente el uso de delantal. Esta indicación, condicionada a cargo -para profesores y asistentes de la educación- y género -mujeres-, presenta manifestaciones de rechazo de generaciones jóvenes. Esta forma de regulación, considerada como una manifestación de disciplinamiento hacia quien es docente, representa una forma de control del cuerpo relacionada con una institucionalidad homogeneizadora y distingue jerarquías ante su uso.

En segundo lugar se identifican regulaciones desde la cultura escolar en el quehacer docente; entre ellas se identifica la planificación clase a clase desde la percepción docente de una acción sin sentido pues la escuela es una organización de cambios constantes en el acontecer cotidiano; exigencias curriculares descontextualizadas a la realidad local; la burocratización de procedimientos cotidianos a partir de la documentalización; supervisión de agentes desde diversas áreas del Ministerio del ramo percibidos desde la desconfianza y generadores de tensión por sus consecuencias y la evaluación docente identificada explícitamente como violenta ante las condiciones en las que se efectúa. Este último elemento se convierte en factor de intensificación laboral al no contemplar los tiempos de trabajo extra que se requieren para cumplir con las exigencias planteadas, lo que obliga a ocupar espacios personales y tiempos de descanso. De este modo, estas acciones se vinculan con el control de la acción pedagógica.

Aquellas acciones, descritas como control de la práctica docente, tienen como base los procesos de rendición de cuentas, realizadas por medio de la documentación de procesos del quehacer cotidiano, supervisiones a la escuela y evaluación docente. Así, la rendición de cuentas exige a la escuela traspasar a documentos protocolos, registros y formularios, cuya ausencia se transforma en una falta grave. Este es un proceso que se posiciona en la escuela generando cambios en la cultura escolar, a partir de la tensión de un saber hacer de manera implícita entre los actores, a la necesidad de tener evidencias. Sin embargo, los tiempos que implican la ejecución de estas acciones no se consideran en la distribución efectiva de las horas para el desarrollo de sus labores cotidianas del profesorado, es decir, en la consideración de sus horas.

La escuela municipal es aquella que evidencia y expone el impacto de las instituciones que supervisan el trabajo en la escuela. Se identifica la distinción de aquellas inspecciones 
cuyas consecuencias pueden ser punitivas o no por medio de la aplicación de multas. Así, tras la visita de la Agencia de Calidad de Educación, se procede a la categorización de colegios, lo que para el establecimiento educacional es visto como "no punitivo" pues no emite multas. Al contrario, las visitas de la Superintendencia de Educación y de Subvención Escolar son definidas como punitivas, al hacer uso de multas ante el error, por ello, genera tensión mayor en la escuela.

En la misma línea, quienes se encargan de la supervisión son definidos como actores que ejercen control, percibidos desde la lógica de la desconfianza frente al trabajo realizado por quienes son actores educativos. Sus visitas generan tensión previa en la comunidad educativa, pues se reiteran indicaciones con el objetivo de evitar sanciones al colegio y la amenaza de castigos -como el registro en la hoja de vida- ante alguna falta o incumplimiento de parte del profesor. Así, profesorado y equipos directivos significan las supervisiones como fuentes de tensión y sobrecarga laboral, teniendo a la base la burocracia, la desconfianza y la visualización de consecuencias para los establecimientos educacionales.

Finalmente se evidencia que en las dos dimensiones analizadas -normativas laborales y cultura escolar-, se presentan diversas formas de control a la base, las que ocurren desde el control del tiempo -principalmente descritas en la dimensión normativa laboral- del cuerpo y de la acción pedagógica -a partir de lo expuesto en cultura escolar-.

\section{DISCUSIÓN Y CONCLUSIONES}

Este estudio se basa en la experiencia en tres establecimientos educacionales de la Región Metropolitana, cada uno de los cuales corresponden a tres formas de dependencia: municipal, particular-subvencionado y particular privado en el momento que está vigente el estatuto docente y el Sistema de Desarrollo Profesional Docente estaba en discusión. Cada una de ellas regula la labor docente desde normativas laborales y desde la cultura escolar. A partir de estos elementos se han evidenciado violencias que emergen en un contexto con reformas donde el neoliberalismo es parte del contexto.

Se evidencia que en las regulaciones de la labor docente -como normativas laborales y cultura escolar- se manifiesta control del tiempo, del cuerpo y de la acción pedagógica. Si se considera la violencia simbólica como un tipo de violencia ausente de dolor visible y legitimada (Crettiez, 2009), que se ejerce sobre el agente social con la anuencia de este, contribuyendo a producir la eficacia de aquello que los determina en la medida en que ellos lo estructuran (Bourdieu y Wacouant, 1995), entonces estas manifestaciones responden a formas de violencia simbólica que afectan en quienes se desempeñan como trabajadores de la educación.

De acuerdo a los resultados y a lo expuesto en la teoría, hay contrastes entre aquello que dictan las normativas laborales que protegen al cuerpo docente como trabajadores, y aquello que se evidencia a partir del trabajo en terreno. Ejemplo de ello es la identificación de tiempos de almuerzo y de horas de trabajo curricular no lectivo que no se han respetado según lo que dicta la ley. A partir de lo anterior, se expone la tensión entre elementos definidos desde las normativas, en interacción con la cultura escolar de cada establecimiento y las dinámicas que favorece cada administración escolar. La relación entre ellas visibiliza y produce violencias, las cuales en esta oportunidad han sido analizadas desde el concepto de violencia simbólica. 
Ahora bien, ante las normas planteadas -principalmente aquellas que emergen desde la cultura escolar- se expone la posición de docentes, la que, si bien en ocasiones es de sumisión y desesperanza, hay quienes plantean manifestaciones de resistencia; algunos lo hacen de manera pasiva, al dejar de seguirlas en el transcurso del tiempo. Por lo demás, se identifica tensión ante las supervisiones externas debido a las consecuencias individuales y al establecimiento, principalmente a partir de multas.

Como consecuencias del contexto descrito, quienes son trabajadores de la educación viven la intensificación laboral (Hargreaves, 2003) y malestar docente (Esteve, 1994), visto desde sus actores bajo el concepto de agobio. Sin embargo, este aspecto no se limita a las condiciones brindadas desde las normas laborales, sino que conviven con una cultura escolar que influye en la forma en que se realiza la labor docente. A ello hay que agregar otros elementos descritos y estudiados, como las condiciones de mercado y competencia del sistema educativo, más las políticas gerencialistas las que afectan el trabajo y subjetividad docente (Oyarzún y Cornejo, 2020), ejemplo de ello son los procesos evaluativos como el portafolio que apunta a la individualidad limitando el trabajo colaborativo efectivo (Ramírez - Casas del Valle; Baleirola y Sisto, 2019) y que a su vez generan la percepción de desconfianza ante el rol desempeñado. Además, las condiciones de financiamiento en las que se sostienen los establecimientos educativos, por medio de los voucher (Cornejo et al., 2015), no solo limitan la gestión administrativa, sino que también la pedagógica.

Este estudio, realizado en el marco de un cambio paradigmático en materia de carrera docente ante el paso de un modelo basado en la experiencia profesional y el perfeccionamiento como mecanismos de reconocimiento profesional (Estatuto Docente) a uno de desempeño asociado a pruebas disciplinares y pedagógicas que repercuten en el encasillamiento (Sistema de Desarrollo Profesional Docente), permite identificar las tensiones que la normativa laboral en interacción con la cultura escolar y la administración educativa producen en la experiencia de los docentes.

Si bien puede ser considerada como una herramienta que favorece o dificulta las condiciones que promueven una mejor educación a partir de la labor docente, la nueva normativa presenta cambios como la definición de actividades curriculares no lectivas denominadas "horas no lectivas", la supervisión de su uso planteado a lo menos un $40 \%$ de la carga horaria, la posibilidad de velar por ella desde los docentes por medio de acciones legales ante el incumplimiento y el financiamiento para las mismas (Mineduc, 2016; CPEIP, 2017), elementos que fueron tratados desde los resultados. Sin embargo, el SDPS, si bien incrementa los sueldos por medio del avance de escalafones, no genera cambios estructurales en los procesos de contratación, institucionalidad, ni cultura; no promueve culturas democráticas y participativas que valoren la práctica pedagógica ni otorgan autonomía al docente para que despliegue sus capacidades pedagógicas que están desvalorizadas por la desconfianza que ha instalado el sistema; y si bien propone la colaboración, más bien refuerza la individualidad. De acuerdo a ello, como proyección, es necesario evidenciar las subjetividades docentes y cambios ocurridos en la implementación de esta nueva normativa en la escuela, que se desarrollan en interacción con las culturas escolares. 


\section{REFERENCIAS BIBLIGRÁFICAS}

Andrade, D. (2006). Trabajo Docente y la Nueva Regulación educativa en América Latina. En Feldfeber, M. y Andrade, D. (2006) Comps. Políticas educativas y trabajo docente. Nuevas regulaciones ¿nuevos sujetos? Noveduc.

Berger, C. y Lisboa, C. (2012). Violencia Escolar. Estudios y Posibilidades de Intervención en Latinoamérica. Editorial Universitaria.

Blase, J. \& Blase, J. (2008). The mistreated teacher: a national study. Journal of Educational Administration, 46(3), 263-301.

Bourdieu, P. y Wacouant, L. (1995). Respuestas. Por una antropología reflexiva. Grijalbo.

Bringiotti, M., Krynveniuk, M. y Lassi, S. (2007). Las múltiples violencias de la «violencia» en la escuela. Hacia un abordaje integrativo del problema. Paidéia, 14(29), 313-325. http://dx.doi.org/10.1590/S0103-863X2004000300007

CPEIP (2017). Orientaciones Sistema de Desarrollo Profesional Docente. CPEIP- MINEDUC.

Cornejo, R. (2008). Entre el Sufrimiento Individual y los Sentidos Colectivos: Salud Laboral Docente y Condiciones de Trabajo. Revista Docencia, 35, 77-85.

Cornejo, R., Albornoz, N., Castañeda, L., Palacios, D., Etcheberrigaray, R., Goméz, S., Hidalgo, F. y Lagos, J. (2015). Las Prescripciones del Trabajo Docente en el Nuevo Marco Regulatorio de Políticas Educativas en Chile. Psicoperspectivas, 14(2), 72-83.

Crettiez, X. (2009). Las Formas de la Violencia. Waldhuter Editores.

Deal, T. \& Peterson, K. (2009). Shaping School Culture: Pitfalls, Paradoxes \& Promises. San Francisco: Josey-Bass.

Denzin, N. y Lincoln, Y. (2012) Introducción general. La investigación cualitativa como disciplina y como práctica. En N. Denzin \& Y. Lincoln (Coords.) Manual de investigación cualitativa Vol. I El campo de la investigación cualitativa (pp. 43-101). Editorial Gedisa.

Dirección del Trabajo (2015). Código del Trabajo. Dirección del Trabajo.

Esteve, J. (1994). El Malestar Docente. Paidós.

Falsarella, A. (2018). Os Estudos sobre a Cultura da Escola: Forma, Tradições, Comunidade, Clima, Participação, Poder. Centro de Estudos Educação e Sociedade - CEDES.

García, M. y Madriaza, P. (2005). Sentido y Propósito de la violencia Escolar. Análisis Estructural del Discurso de Estudiantes Agresivos del Nivel Socioeconómico Alto. Revista de Psicología de la Universidad de Chile, XIV(1), 41- 59.

Geertz, C. (2005). La Interpretación de las Culturas. Gedisa.

Govea, V.; Vera, G. y Vargas, A. (2011). Etnografía: una mirada desde corpus teórico de la investigación cualitativa Omnia, 17(2), 26-39.

Hargreaves, A. (2003). Profesorado, Cultura y Postmodernidad. Morata.

Köhler, H. y Artiles, A. M. (2010). Manual de la Sociología del Trabajo y de las Relaciones Laborales. Delta Publicaciones.

Mayring, P. (2014). Qualitative content analysis: theoretical foundation, basic procedures and software solution. SSOAR. Extraído de URN: http://nbn-resolving.de/urn:nbn:de:0168-ssoar-395173

Mineduc. (1997). Ley 19.070. Fija Texto Refundido, Coordinado Y Sistematizado De La Ley N ${ }^{\circ}$ 19.070 que Aprobó el Estatuto de dos Profesionales de la Educación, y de las Leyes que la Complementan y Modifican. Biblioteca del Congreso Nacional de la República de Chile. (22 de enero de 1997).

(2005). Principales Resultados del Estudio Nacional de Violencia en el Ámbito Escolar. Ministerio de Educación.

. (2011). Ley 20.501. Calidad y Equidad de la Educación. Biblioteca del Congreso Nacional de la República de Chile. Santiago, Chile. (26 de Febrero de 2011).

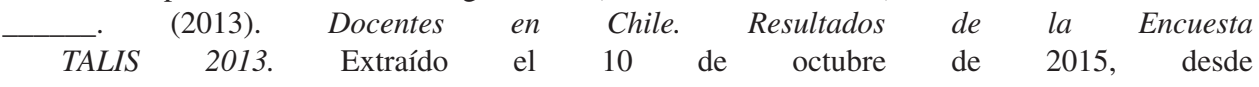


http://centroestudios.mineduc.cl/tp_enlaces/portales/tp5996f8b7cm96/uploadImg/File/ Evidencias/evidencia_final_marzo_2015.pdf

. (2016). Ley 20.903. Crea el Sistema de Desarrollo Profesional Docente y Modifica otras Normas. Biblioteca del Congreso Nacional de la República de Chile. (1 de abril de 2016).

Morales, M., Álvarez, J. P., Ayala, Á., Ascorra, P., Bilbao, M., Carrasco, C., López, V., Olavarría, D., Ortiz, S., Urbina, C. y Villalobos, B. (2014). Violencia Escolar a Profesores: Conductas de Victimización Reportadas por Docentes de Enseñanza Básica. Revista de Estudios Cotidianos - NESOP, 2, 91-116.

OECD (2020). Notas País. Resultados Talis 2018. Volumen II. OECD Publishing

Oliveira, D., Goncalves, G., Melo, S. (2004). Cambios en la Organización del Trabajo Docente, Consecuencias para los Profesores. RMIE, 9(20), 183-197.

Ortiz-Molina, B. (2011). Violencia escolar: la mirada de los maestros sobre las relaciones de colegaje. Magis, 4, 369-382.

Oyarzún, C. y Cornejo, R. (2020). Trabajo docente y nueva gestión pública en Chile: una revisión de la evidencia. Educ. Soc., Campinas, 41, 1-24. https://doi.org/10.1590/ES.219509

Ramírez - Casas del Valle, L., Baleirola, E. y Sisto, V. (2019). Pedagogía en la Era de la Rendición de cuentas: Análisis del Portafolio Docente en Chile. Educ. Soc., Campinas, 40. 